\title{
Relaxation response of polymers containing highly flexible side groups monitored by broadband dielectric spectroscopy
}

\author{
Cristina Alvarez and Vicente Lorenzo \\ Escuela Técnica Superior de Ingenieros Industriales (UPM), 28006 Madrid, Spain \\ Evaristo Riande ${ }^{\text {a) }}$ \\ Instituto de Ciencia y Tecnología de Polímeros (CSIC), 28006 Madrid, Spain
}

(Received 27 December 2004; accepted 9 March 2005; published online 16 May 2005)

\begin{abstract}
The relaxation behavior of poly(5-acryloxymethyl-5-methyl-1,3-dioxacyclohexane), a polymer containing highly flexible side groups, is studied by broadband dielectric spectroscopy in the frequency and temperature ranges $10^{-1}-10^{9} \mathrm{~Hz}$ and $123-473 \mathrm{~K}$, respectively. Above the glass transition temperature $T_{g}$ the dielectric loss in the frequency domain exhibits a prominent $\alpha$ absorption, followed in increasing order of frequencies by two secondary absorptions called $\beta$ and $\gamma$. At temperatures slightly higher than $T_{g}$, the $\alpha$ relaxation is well separated from the $\beta$, but as temperature increases overlapping between both relaxations augments forming an $\alpha \beta$ absorption in the vicinity of $420 \mathrm{~K}$. This latter absorption displays a shoulder on its high-frequency side corresponding to the $\gamma$ relaxation. The strength of the $\alpha$ relaxation decreases with increasing temperature, eventually vanishing at the temperature at which the $\alpha \beta$ absorption is formed. The time retardation spectra of the isotherms are calculated and further used to facilitate the deconvolution of the overlapping relaxations. The fact that the temperature dependence of the $\beta$ relaxation also describes that of the $\alpha \beta$ absorption suggests that both relaxations have the same nature. It seems that as temperature increases, the $\alpha$ relaxation feeds on the $\beta$ absorption until its complete disappearance. The $\gamma$ relaxation, in turn, seems to increase at the expense of the $\alpha \beta$ process at high temperature. (c) 2005 American Institute of Physics. [DOI: 10.1063/1.1900064]
\end{abstract}

\section{INTRODUCTION}

Above the glass transition temperature $T_{g}$ in the moderate supercooled regime, liquids exhibit a single absorption. In cooling these substances, a temperature is reached at which the single absorption splits into a slow relaxation, called $\alpha$, and a fast one, named $\beta$. ${ }^{1}$ The distance between the peaks of the two relaxations in the frequency domain increases as temperature decreases until ergodicity is lost and a transition from the liquid (rubbery) state to the glassy state occurs. ${ }^{2}$ Motions associated with the $\alpha$ relaxation freeze at the glass transition temperature, while the $\beta$ relaxation remains operative in the glassy state. The mean-relaxation time associated with the $\alpha$ relaxation undergoes an anomalous increase in the vicinity of $T_{g}$ described by the empirical Vogel-Fulcher-Tammann-Hesse (VFTH) equation ${ }^{3-5}$

$$
\tau=\tau_{0} \exp \left(\frac{m}{T-T_{V}}\right),
$$

where $\tau_{0}$ is a prefactor of the order of picoseconds and $T_{V}$ is the Vogel temperature, where the extrapolated relaxation time diverges. ${ }^{2,6}$ The fact that Eq. (1) also fits the temperature dependence of the viscosity suggests that the structural or $\alpha$ relaxation is a precursor of both the glassy state and the viscous flow.

The study of the dynamic behavior of both the glassy state and its precursor, the supercooled liquid, is paramount

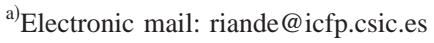

to obtain a full understanding of the glass transition, one of the most important unresolved issues in condensed matter physics. $^{2,3,6,7}$ The rather low sensitivity of the glass-rubber relaxation of high-molecular-weight polymers to chain length, suggests that the $\alpha$ relaxation arises from cooperative micro-Brownian motions. However, it is still necessary to establish the temperature and frequency at which the cooperative $\alpha$-dynamic relaxation sets in as a function of the chemical structure and the different scenarios for the separation of the $\alpha$ and $\beta$ relaxations.

Polymers with cyclohexyl or 1,3-dioxacyclohexyl side groups are characterized for displaying considerable dielectric and mechanical activity in the glassy state. For example, the isochrones representing the temperature dependence of the loss of poly(cyclohexyl acrylate) exhibit a prominent $\beta$ absorption in the vicinity of $193 \mathrm{~K}$ at $1 \mathrm{~Hz} .^{8}$ In the same way, the isochrones of poly(5-acryloxymethyl-5-ethyl-1,3dioxacyclohexane) (PAMED) also present a prominent $\beta$ relaxation in the glassy state. ${ }^{9}$ The intensity of this relaxation severely diminishes if a phenyl group replaces the hydrogen atom of the 1,3-dioxacyclohexyl group bonded to the carbon atom in position 2. ${ }^{10}$ Therefore this sort of polymer is especially amenable for the study of chain dynamics above and below the glass transition temperature. The polymer chosen in this study is poly(5-acryloxymethyl-5-methyl-1,3dioxacyclohexane) (PAMMD). Isochrones showing the mechanical and dielectric behavior of this polymer in the glassy state were reported elsewhere. ${ }^{11}$ Here we report dielectric results in the frequency domain for PAMMD obtained over a 


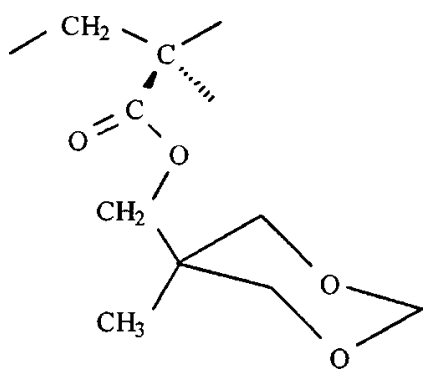

FIG. 1. Scheme of the repeating unit of poly(5-acryloxymethyl-5-methyl1,3-dioxacyclohexane) (PAMMD).

wide temperature window from which the time retardation spectra at different temperatures were calculated. The spectra were further used to deconvolute overlapping peaks involved in the response of the system to electric-field perturbations, thus facilitating the interpretation of the dielectric results. Special attention is paid to the merging of the $\alpha$ and $\beta$ relaxations to form the $\alpha \beta$ absorption. The temperature dependence of the relaxation times of the $\alpha, \beta, \alpha \beta$, and $\gamma$ processes are also investigated.

\section{EXPERIMENTAL PART}

Poly(5-acryloxymethyl-5-methyl-1,3-dioxacyclohexane) was synthesized by radical polymerization of 5-acryloxymethyl-5-methyl-1,3-dioxacyclohexane in toluene solution. The polymerization was carried out at $323 \mathrm{~K}$, in nitrogen atmosphere, using azo-bis-isobutyronitrile as initiator. A description of the synthesis of both monomer and polymer is given elsewhere. ${ }^{11}$ A scheme of the repeating unit of PAMMD is shown in Fig. 1.

The glass transition temperature $T_{g}$ was determined with a Perkin-Elmer DSC7 apparatus at a constant heating rate of $20 \mathrm{~K} / \mathrm{min}$, under nitrogen atmosphere. The sample was heated twice and $T_{g}$, taken as the temperature corresponding to the middle point of the endothermic step during the second scan, was $313 \mathrm{~K}$.

Complex dielectric permittivity measurements were performed over a broad frequency window, $10^{-1}-10^{9} \mathrm{~Hz}$, in the temperature range $123-473 \mathrm{~K}$. To cover the ten-decades frequency window, two different experimental setups were used: a Novocontrol (Huntsangen, Germany) system integrating a SR 830 lock-in amplifier with an ALPHA dielectric interface was employed in the $10^{-1}-10^{7}-\mathrm{Hz}$ range, while the dielectric measurements in the $10^{6}-10^{9}-\mathrm{Hz}$ range were obtained by means of a HP4291 (Hewlet Packard, Hyogo, Japan) coaxial line reflectometer. In this case, the complex permittivity was determined by measuring the reflection coefficient at a particular reference plane. ${ }^{12}$ These systems were integrated in a Novocontrol broadband dielectric spectrometer. The temperature was controlled by nitrogen jet (QUATRO from Novocontrol) with a temperature error of $\approx 0.1 \mathrm{~K}$ during every single sweep in frequency. The samples were prepared by pressing the melt between two thin aluminum electrodes separated by a 0.12 -mm-thick circular kapton spacer. Previously the polymer was dried overnight at $333 \mathrm{~K}$ in vacuum. A kapton spacer was used to control the sample thickness and to avoid short-circuit of the two electrodes.

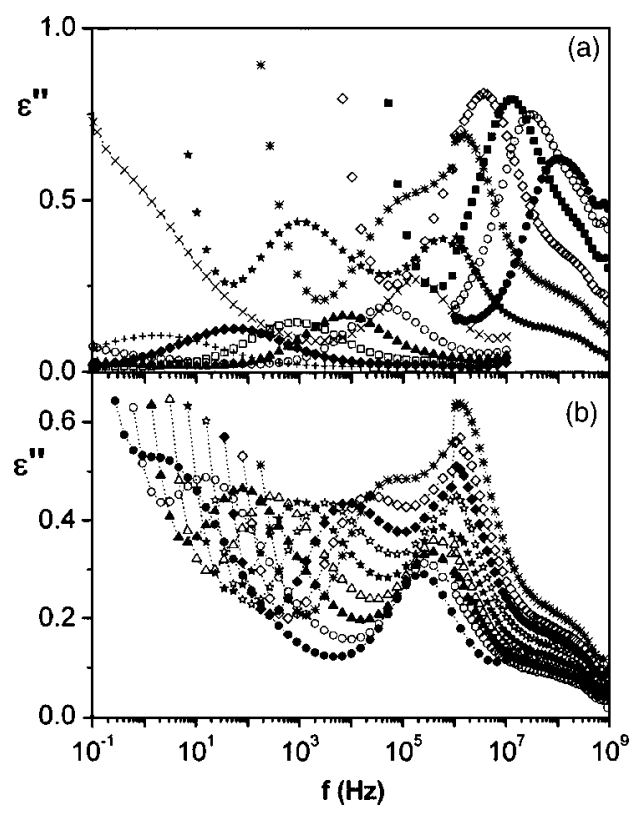

FIG. 2. (a) Dielectric loss isotherms in the frequency domain at several temperatures covering the glassy, rubbery, and flow regions: $(+) 198 \mathrm{~K},(\bullet)$ $223 \mathrm{~K},(\square) 248 \mathrm{~K},(\boldsymbol{\Delta}) 273 \mathrm{~K},(\bigcirc) 298 \mathrm{~K},(\times) 323 \mathrm{~K},(\star) 348 \mathrm{~K}$, (*) 373 $\mathrm{K},(\diamond) 398 \mathrm{~K},(\boldsymbol{\square}) 423 \mathrm{~K},(\odot) 448 \mathrm{~K},(\bigcirc) 473 \mathrm{~K}$. (b) Isotherms in the rubbery state from 328 to $368 \mathrm{~K}$ at intervals of $5 \mathrm{~K}$.

Samples with 2- and 0.5-cm diameter were utilized for measurements carried out at $f<10^{6} \mathrm{~Hz}$ and $f>10^{6} \mathrm{~Hz}$, respectively. The dielectric measurements obtained in the $10^{6}-10^{9}-\mathrm{Hz}$ range were corrected by a geometry factor of 1.05 .

\section{RESULTS}

Illustrative curves showing the dielectric loss in the frequency domain at several temperatures are represented in Fig. 2. The isotherms exhibit a well-developed peak below $T_{g}$ associated with the $\beta$ relaxation. On the high-frequency side of this absorption a second relaxation seems to begin to show. Above $T_{g}$ the isotherms display an ostensible $\alpha$ relaxation, followed at higher frequencies by the $\beta$ process that presents a shoulder at its high-frequency side corresponding to a weak secondary absorption named $\gamma$. To test the reliability of the $\gamma$ absorption, dielectric loss curves were also obtained as a function of temperature at $T<T_{g}$ and the pertinent isochrones at several frequencies are represented in the inset of Fig. 3. The isochrones present a $\beta$ peak the location of which in the spectra shifts, as usual, to higher temperatures with increasing frequency. An attentive inspection of Fig. 3 shows the presence of the $\gamma$ process, which presumably corresponds to the shoulder appearing in the highfrequency side of the isotherms at $T \gg T_{g}$.

At temperatures slightly higher than $T_{g}$ the contribution of the ionic conductivity to the loss is dominant at low frequencies and the $\alpha$ relaxation is not well defined. This contribution scales as $\varepsilon^{\prime \prime} \sim f^{s}$, where $s$ is a parameter close to unity (in fact, $s$ is the unity in pure ionic conductivity), and this contribution becomes negligible at high frequencies. As the temperature of the isotherms increases, the loss curves exhibit two well-defined peaks corresponding to the $\alpha$ relax- 


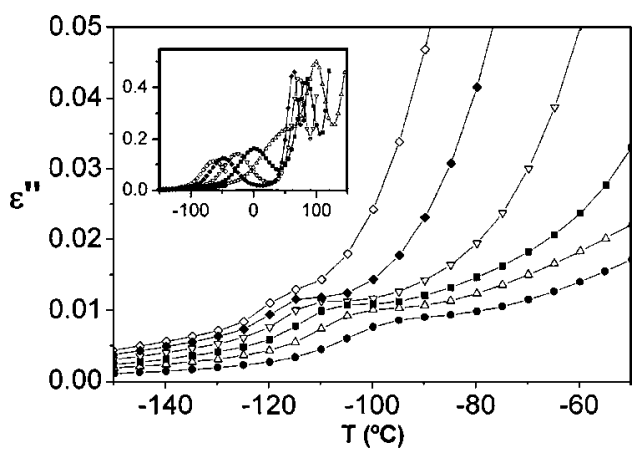

FIG. 3. Isochrones at low temperatures: $(\diamond) 6.87 \mathrm{~Hz},(\diamond) 5.22 \times 10^{1} \mathrm{~Hz}$, $(\nabla) 5.94 \times 10^{2} \mathrm{~Hz}, \quad(\square) 6.77 \times 10^{3} \mathrm{~Hz},(\triangle) \quad 7.71 \times 10^{4} \mathrm{~Hz},(\bullet) \quad 8.78$ $\times 10^{5} \mathrm{~Hz}$. Inset: the same isochrones in the whole temperature window.

ation at low frequencies and the $\beta$ relaxation at higher frequencies. The intensity of the $\beta$ peak increases as temperature augments. As usual, the locations of the $\alpha$ and $\beta$ peaks shift to higher frequencies with increasing temperature. However, owing to its high activation energy, the $\alpha$ relaxation moves faster than the $\beta$ until eventually $\alpha$ reaches $\beta$, becoming a single peak named $\alpha \beta$ absorption in the vicinity of $420 \mathrm{~K}$.

\section{Peaks deconvolution: Retardation spectra}

To deconvolute the overlapping peaks in the isotherms of Fig. 2 some considerations have to be made. In general, symmetric secondary absorptions are described by the Fuoss-Kirkwood equation ${ }^{13}$

$$
\varepsilon^{\prime \prime}(\omega)=\frac{2 \varepsilon_{\max }^{\prime \prime}\left(\omega / \omega_{\max }\right)^{m}}{1+\left(\omega / \omega_{\max }\right)^{2 m}},
$$

where $m$ is an empirical parameter the value of which lies in the interval $0<m \leqslant 1$ and $\omega_{\max }$ is the frequency at the maximum of the peak at which the loss is $\varepsilon_{\max }^{\prime \prime}$. The broader the peak, the lower $m$ is, and for a Debye-type peak $m=1$. According to the linear theory of dielectrics, the strength of secondary relaxations is given by

$$
\varepsilon_{r}-\varepsilon_{u}=\frac{2}{\pi} \int_{-\infty}^{\infty} \varepsilon^{\prime \prime}(\omega) d \ln \omega .
$$

Substituting Eq. (2) into Eq. (3) yields

$$
\varepsilon_{r}-\varepsilon_{u}=\frac{2 \varepsilon_{\max }^{\prime \prime}}{m},
$$

where $\varepsilon_{r}$ and $\varepsilon_{u}$ are, respectively, the relaxed and unrelaxed dielectric permittivities associated with the absorption. On the other hand, the decay of the $\alpha$ relaxation in the time domain is inevitably described by a Kohlrausch-WilliamsWatts $(\mathrm{KWW})^{14,15}$ type stretched exponential equation

$$
\phi(t)=\exp \left[-\left(\frac{t}{\tau_{\mathrm{KWW}}}\right)^{\beta_{\mathrm{KWw}}}\right],
$$

where $0<\beta_{\mathrm{KWW}} \leqslant 1$. For a Debye-type peak, $\beta_{\mathrm{KWW}}=1$. Transformation of the dielectric permittivity from the time domain to the frequency domain gives ${ }^{16}$

$$
\frac{\varepsilon^{*}(\omega)-\varepsilon_{u}}{\varepsilon_{r}-\varepsilon_{u}}=1-i \omega \int_{0}^{\infty} \phi(t) \exp (-i \omega t) d t .
$$

Therefore the dielectric loss can be written as

$$
\begin{aligned}
\varepsilon^{\prime \prime}(\omega)= & \left(\frac{\sigma}{\varepsilon_{0} \omega}\right)^{s}+\left(\varepsilon_{r}-\varepsilon_{u}\right)_{\alpha} \operatorname{Im}[i \omega F(\phi(t))] \\
& +\frac{2 \varepsilon_{\max , \beta}^{\prime \prime}\left(\omega / \omega_{\max , \beta}\right)^{m_{\beta}}}{1+\left(\omega / \omega_{\max , \beta}\right)^{2 m_{\beta}}}+\frac{2 \varepsilon_{\max , \gamma}^{\prime \prime}\left(\omega / \omega_{\max , \gamma}\right)^{m_{\gamma}}}{1+\left(\omega / \omega_{\max , \gamma}\right)^{2 m_{\gamma}}},
\end{aligned}
$$

where Im means the imaginary part of the quantity between brackets, $F$ is the Fourier transform, and $\varepsilon_{0}$ is the dielectric permittivity in vacuum. The first term on the right-hand side of Eq. (6) is the contribution of the conductivity to the loss, whereas the second term can be obtained by the seriesexpansion method proposed by Williams et al. ${ }^{17}$

$$
\begin{aligned}
\frac{\varepsilon^{*}(\omega)-\varepsilon_{r}}{\varepsilon_{u}-\varepsilon_{r}}= & \sum_{n=1}^{\infty} \frac{(-1)^{n-1}}{\left(\omega \tau_{\mathrm{KWW}}\right)^{n \beta_{\mathrm{KWW}}}} \frac{\Gamma\left(n \beta_{\mathrm{KWW}}+1\right)}{\Gamma(n+1)} \\
& \times \exp \left(i n \beta_{\mathrm{KWW}} \frac{\pi}{2}\right), \quad \omega \tau_{\mathrm{KWW}}>0 \\
\frac{\varepsilon^{*}(\omega)-\varepsilon_{r}}{\varepsilon_{u}-\varepsilon_{r}}= & \sum_{n=1}^{\infty}(-1)^{n-1} \frac{\left(\omega \tau_{\mathrm{KWW}}\right)^{n-1}}{\Gamma(n)} \Gamma\left(\frac{n+\beta_{\mathrm{KWW}}-1}{\beta}\right) \\
& \times \exp \left(\frac{i \pi(n-1)}{2}\right), \quad \omega \tau_{\mathrm{KWW}} \rightarrow 0,
\end{aligned}
$$

where $\Gamma$ is the Euler gamma function.

The complex dielectric permittivity corresponding to the $\alpha$ relaxation in the frequency domain can also be described by the Havriliak-Negami (HN) equation ${ }^{18}$

$$
\varepsilon^{*}(\omega)=\varepsilon_{u}+\frac{\left(\varepsilon_{r}-\varepsilon_{u}\right)}{\left[1+\left(i \omega \tau_{\mathrm{HN}}\right)^{a}\right]^{b}} .
$$

The HN equation contains two shape parameters $a$ and $b$, i.e., one more than the KWW equation. The exponents $a$ and $b(0 \leqslant a, a b \leqslant 1)$ are related to the width and asymmetry of the relaxation. It is worth noting that the $\mathrm{HN}$ parameters usually found are correlated in such a way that the HN equation corresponds to the KWW equation in the time domain. ${ }^{19}$ Secondary relaxations in the frequency domain are well described by the HN equation with $b=1$. They are symmetric in the logarithmic frequency domain and present a maximum at $\omega_{\max }=1 / \tau_{\mathrm{HN}}$. This symmetric HN function is known as the Cole-Cole equation. For the asymmetric $\alpha$ relaxation, $\omega_{\max } \tau_{\mathrm{HN}}>1$. The dielectric loss is given by

$$
\frac{\varepsilon^{\prime \prime}(\omega)}{\varepsilon_{r}-\varepsilon_{u}}=-\operatorname{Im}\left\{\left[1+\left(i \omega \tau_{\mathrm{HN}}\right)^{a}\right]^{-b}\right\} .
$$

The loss curves described by Eq. (9) display a maximum at a frequency $\omega_{\max }$ given by ${ }^{20,21}$

$$
\omega_{\max }=\frac{1}{\tau_{\mathrm{HN}}}\left[\sin \left(\frac{\pi a b}{2(b+1)}\right) / \sin \left(\frac{\pi a}{2(b+1)}\right)\right] .
$$

Using the $\mathrm{HN}$ formalism, the dielectric loss can be written as 


$$
\begin{aligned}
\varepsilon^{\prime \prime}(\omega)= & \left(\sigma / \varepsilon_{0} \omega\right)^{s}-\left(\varepsilon_{r}-\varepsilon_{u}\right)_{\alpha} \operatorname{Im}\left\{\left[1+\left(i \omega \tau_{\mathrm{HN}, \alpha}\right)^{a} \alpha\right]^{-b}\right\} \\
& -\left(\varepsilon_{r}-\varepsilon_{u}\right)_{\beta} \operatorname{Im}\left\{\left[1+\left(i \omega \tau_{\mathrm{HN}, \beta}\right)^{a_{\beta}}\right]^{-1}\right\} \\
& -\left(\varepsilon_{r}-\varepsilon_{u}\right)_{\gamma} \operatorname{Im}\left\{\left[1+\left(i \omega \tau_{\mathrm{HN}, \gamma}\right)^{a_{\gamma}}\right]^{-1}\right\} .
\end{aligned}
$$

For reasons that will become clear later on, deconvolutions of the overlapping peaks are better accomplished from retardation spectra. According to the phenomenological theory of linear dielectrics, the global response of amorphous condensed systems to perturbation fields can be expressed in terms of a continuous distribution of independent Debye relaxations. The density function of this distribution is known as the non-normalized retardation time spectrum $L(\tau)$ in such a way that the imaginary part of the dielectric permittivity can be expressed in terms of $L(\tau)$ as ${ }^{22}$

$$
\varepsilon^{\prime \prime}(\omega)=\left(\frac{\sigma}{\varepsilon_{0} \omega}\right)^{-s}+\int_{-\infty}^{\infty} L(\log \tau) K(\omega, \tau) d \log \tau,
$$

where $K(\omega, \tau)=\omega \tau /\left(1+\omega^{2} \tau^{2}\right)$ is the kernel of this equation, $\sigma$ is the electrical conductivity, and $\varepsilon_{0}$ is the permittivity in the vacuum. The nonlinear dependence of the conductivity term on $s$, one of the parameters to be estimated, introduces a supplementary complexity to the inversion of Eq. (12) to determine $L(\log \tau)$. Obtaining different $L$ 's for different physically admissible values of $s$ lying in the range $0.8-1$ and selecting the one that optimizes the approximation circumvent this difficulty. This procedure transforms the initial nonlinear problem into another that is linear, i.e., into solving a first-kind Fredholm integral equation. Unfortunately the inversion of Eq. (12) is usually ill-posed and, consequently, it is necessary to use some special procedures to obtain $L(\log \tau)$ and $\sigma$.

Let us assume that we want to calculate the spectrum for some discrete times $L\left(\log \tau_{\mathrm{j}}\right),(j=1,2, \ldots, m-1)$ and $\sigma$ from a set of $n$ measurements of $\varepsilon^{\prime \prime}\left(\omega_{i}\right)$ at angular frequencies $\omega_{i}(i=1,2, \ldots, n)$ with uncertainties $\rho_{i}$. Then Eq. (12) can be rewritten in the form

$$
\frac{\varepsilon^{\prime \prime}\left(\omega_{i}\right)}{\sigma_{i}} \cong\left[\left(\frac{\sigma}{\varepsilon_{0} \omega_{i}}\right)^{-s}+\sum_{k=1}^{m} R_{i k} L\left(\log \tau_{k}\right)\right] / \rho_{i},
$$

where $R_{i k}$ reads for

$$
R_{i k}=K\left(\omega_{i}, \tau_{k}\right) \log \left(\frac{\tau_{k+1}}{\tau_{k-1}}\right)^{1 / 2} .
$$

Equation (13) can be written in terms of the matrix notation as

$$
\left[\begin{array}{c}
\varepsilon^{\prime \prime}\left(\omega_{1}\right) / \rho_{1} \\
\varepsilon^{\prime \prime}\left(\omega_{2}\right) / \rho_{2} \\
\ldots \\
\varepsilon^{\prime \prime}\left(\omega_{n}\right) / \rho_{n}
\end{array}\right] \cong\left[\begin{array}{ll}
R_{11} / \rho_{1} & \mathbf{R}_{12} / \rho_{1} \ldots \mathbf{R}_{1 m-1} / \rho_{1} \omega_{1}^{-s} / \rho_{1} \\
R_{21} / \rho_{2} & \mathbf{R}_{22} / \rho_{2} \ldots \mathbf{R}_{2 m-1} / \rho_{2} \omega_{2}^{-s} / \rho_{2} \\
\ldots \\
R_{n 1} / \rho_{n} & \mathbf{R}_{n 2} / \rho_{n} \ldots \mathbf{R}_{n m-1} / \rho_{n} \omega_{n}^{-s} / \rho_{n}
\end{array}\right] \cdot\left[\begin{array}{c}
L\left(\tau_{1}\right) \\
L\left(\tau_{2}\right) \\
\ldots \\
L\left(\tau_{m-1}\right) \\
\left(\frac{\sigma}{\varepsilon_{0}}\right)^{-s}
\end{array}\right]
$$

or in a more compact form:

$$
\varepsilon^{\prime \prime} \cong \mathbf{R} \mathbf{L} .
$$

The solution of the ill-conditioned Eq. (15) is similar to that of the also ill-posed quadratic programming problem $\min \left|\boldsymbol{\varepsilon}^{\prime \prime}-\mathbf{R} \mathbf{L}\right|^{2}$, equivalent to ${ }^{23}$

$$
\min \left|\boldsymbol{\varepsilon}^{\prime \prime}-\mathbf{R} \mathbf{L}\right|^{2}+\lambda\left(\mathbf{L}^{T} \mathbf{H L}\right),
$$

where $\lambda(>0)$ is known as the regularization parameter and $\mathbf{H}$ is a definite positive quadratic form. The main idea underlying this transformation is that when a quadratic minimization principle is combined with a quadratic constraint, and both are positive, only one of the two needs to be nondegenerate for the overall problem to be well-posed.

The election of the $\mathbf{H}$ matrix must be based on the $a$ priori knowledge of the solution. If the solution is thought to be piecewise linear, a good choice is $\mathbf{H}=\mathbf{B}^{T} \mathbf{B}$, where $\mathbf{B}$ is the $(m-2) \times m$ matrix: ${ }^{23}$

$$
\mathbf{B}=\left[\begin{array}{rrrrrrr}
-1 & 2 & -1 & 0 & 0 & \ldots & 0 \\
0 & -1 & 2 & -1 & 0 & \ldots & 0 \\
\ldots & & & & & & \\
0 & \ldots & & -1 & 2 & -1 & 0 \\
0 & \ldots & & 0 & -1 & 2 & -1
\end{array}\right]
$$

and $\mathbf{B}^{T}$ reads for the transpose matrix of $\mathbf{B}$. The solutions corresponding to different values of the regularization parameter $\lambda$ were obtained by means of an iterative technique (conjugated gradient) that allows the utilization of the projection onto convex sets (POCS) methods for achieving a non-negative solution. The best values of $\lambda$ were determined by means of two different procedures: the Morozov's discrepancy method ${ }^{24}$ and the $L$-curve procedure. ${ }^{25}$ The agreement between the retardation time spectra obtained by both methods was rather good, though the loss curves obtained from the spectra calculated using the discrepancy method fit somewhat better the experimental results than the alternative $L$-curve method. Therefore, the retardation spectra appearing in subsequent figures were obtained by the discrepancy 

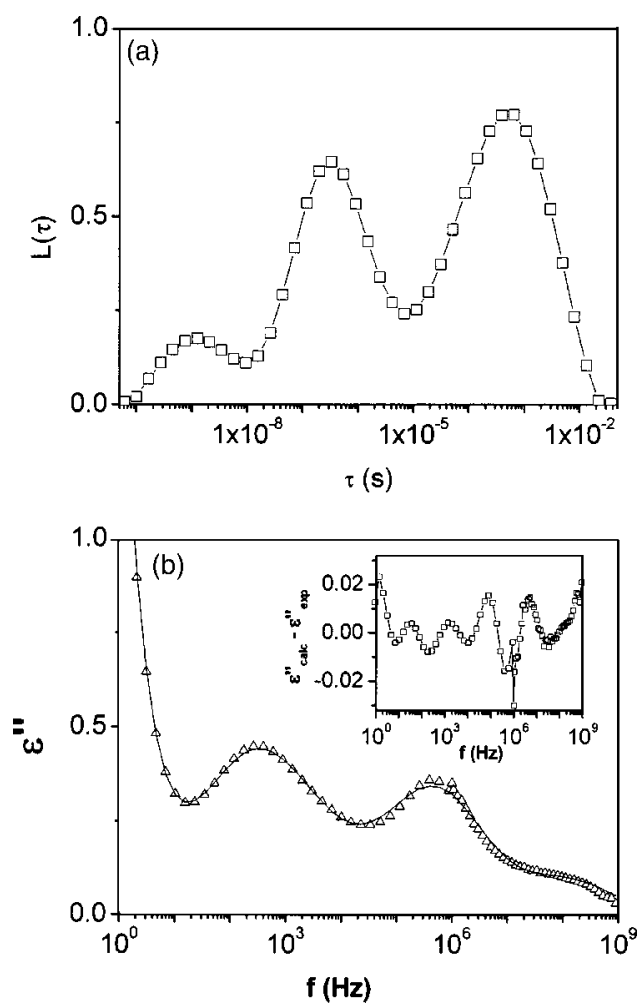

FIG. 4. (a) Non-normalized retardation time spectra $L(\tau)$ obtained from the 343-K measurements with regularization parameters, determined by the discrepancy method. (b) Comparison between the experimental values of the dielectric loss permitivitty $\varepsilon^{\prime \prime}$ measured at $343 \mathrm{~K}(\triangle)$ and the values calculated from the spectrum determined by the discrepancy method (continuous line). The inset shows the residuals for the fit.

method. For illustrative purposes, the retardation time spectrum at $343 \mathrm{~K}$ together with the dielectric loss in the frequency domain obtained from the spectrum are shown in Figs. 4(a) and 4(b), respectively. It can be seen that the values of $\varepsilon^{\prime \prime}$ calculated from the spectrum fit pretty well to the experimental results, observing that the accuracy of the retardation spectrum decreases in the region where data obtained from different instruments intersect. The retardation time spectrum in Fig. 4(a) shows two symmetric peaks together with an asymmetric peak, which are more clearly resolved than those of the $\varepsilon^{\prime \prime}(\omega=2 \pi f)$ curve. As expected, the positions of the maxima of these peaks, $\tau_{\max }$, are related to those of the $\varepsilon^{\prime \prime}$ versus $\omega$ curve by means of the expression $\omega_{\max } \tau_{\max } \cong 1$.

It is important to remark that the $\tau$ scale covers more decades than the $\omega$ scale, as a consequence of the fact that each value of $\varepsilon^{\prime \prime}(\omega)$ is determined from those of the function $L$ in an interval with center in $1 / \omega$. In fact, we have calculated $L$ for $\tau$ values that are two decades above and below the $1 / \omega$ experimental range. Because the intensity of Debye relaxations falls to $1 \%$ of its maximum value for $\omega \tau=10^{ \pm 2}$, the contributions to the experimental results of the hypothetical relaxations that are two decades apart from the interval of interest can be neglected. It is important to remark that this modus operandi that allows minimizing aliasing effects does not require any assumptions about the values of $\varepsilon^{\prime \prime}$ out of the experimental window. Obviously, the values of the spectrum that are closer to the extremes of its interval of definition are less accurate than those of the middle because the former not only depend on the experimental results but also depend on values of $\varepsilon^{\prime \prime}$ at frequencies that have not been accessed during the experiments. The extreme values are totally accurate if $\varepsilon^{\prime \prime}$ vanishes out of the $\tau^{-1}$ range, a condition that is presumably fulfilled by most of our experiments after subtracting the conductivity contribution.

Although the use of the retardation time spectra to deconvolute overlapping relaxations seems to be a complex process, the superposition of several phenomenological functions often utilized for this purpose presents some disadvantages. One of these is the need for fixing the number of processes involved in the loss curves as well as the symmetric or asymmetric character of the peaks. Another disadvantage is related to the difficulty of distinguishing between processes the retardation times of which are close. In contrast, the number of individual processes controlling the overall relaxation is not an a priori assumption for the retardation time spectrum calculation. Even more, there is no need for using different expressions to analyze symmetric and asymmetric peaks. Also, the ability to resolve two processes with comparable retardation times is higher in the spectrum than in the imaginary part of the permittivity curves because the peaks associated with each process are narrower in the former. This extreme can easily be understood if we consider that the Debye relaxation in the time domain is a Dirac delta function, whereas the half-width of the relaxation in the frequency domain is slightly greater than one decade. As a consequence of these and other advantages, the retardation-timespectra formalism has previously been used to analyze dielectric relaxation spectroscopy results by using the pro-

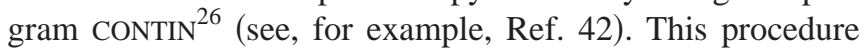
has some drawbacks when compared with that reported in this paper that are related to the way of dealing with the conductivity contribution, the determination of a nonaliased solution, the method that is used to determine the regularization parameter, and the computational efficiency of the general methods for solving quadratic programming problems.

The retardation time spectra obtained at temperatures below $T_{g}$, between $T_{g}$ and the $\alpha \beta$ merging temperature $T_{\text {me }}$, and at $T>T_{\text {me }}$ are represented in Fig. 5. Figure 5(a) exhibits sub- $T_{g}$ spectra that display two symmetric peaks related to the $\beta$ and $\gamma$ relaxations. As shown in Fig. 5(b), an asymmetric peak connected with the $\alpha$ relaxation appears when the temperature rises above $T_{g}$. The $\alpha$ peak shifts to lower times more rapidly than $\beta$ as a result of the high activation energy of the $\alpha$ peak. The intensity of this latter peak decreases as it approaches the $\beta$ peak the intensity of which, in turn, increases. For temperatures close to $420 \mathrm{~K}$, both relaxations merge into a single absorption, known as $\alpha \beta$ relaxation. The spectra at temperatures above $420 \mathrm{~K}$ only exhibit the $\alpha \beta$ and the $\gamma$ peaks [Fig. 5(c)]. It can be argued that the $\gamma$ maximum appears at $\tau$ values that are lower than the inverse of the higher experimental angular frequency for temperatures greater than $423 \mathrm{~K}$ and that the high-frequency side of this relaxation is out of the experimental window. However, an inspection of Fig. 9 clearly shows that these data fit the same line as the more reliable ones obtained at lower temperatures.

The deconvolutions of the sub- $T_{g} \beta$ and $\gamma$ peaks were 

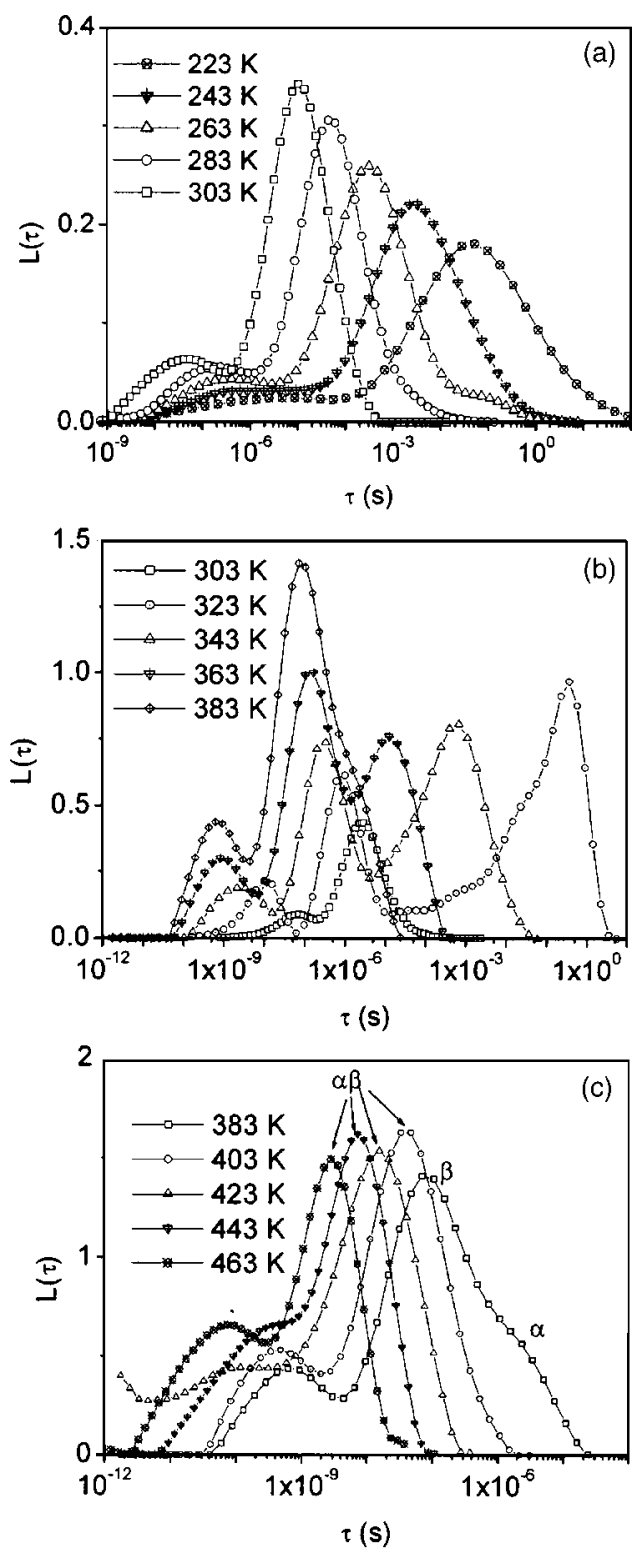

FIG. 5. Non-normalized retardation time spectra $L(\tau)$ calculated from the dielectric relaxation experimental data that were obtained at sub- $T_{g}$ temperatures (a), between $T_{g}$ and the merging temperature (b), and above the merging temperature (c)

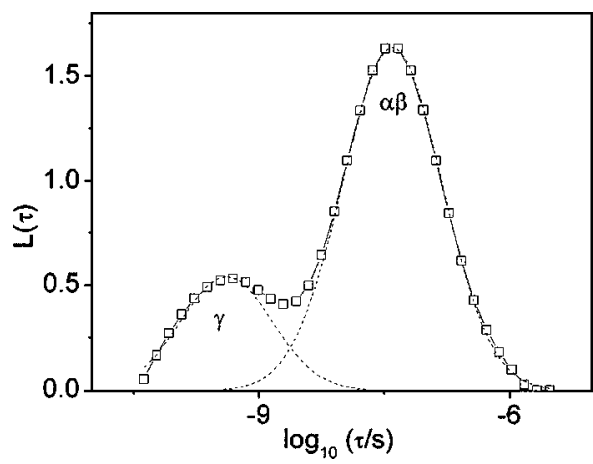

FIG. 6. Deconvolution of the non-normalized retardation time spectrum $L(\tau)$ at $403 \mathrm{~K}$.

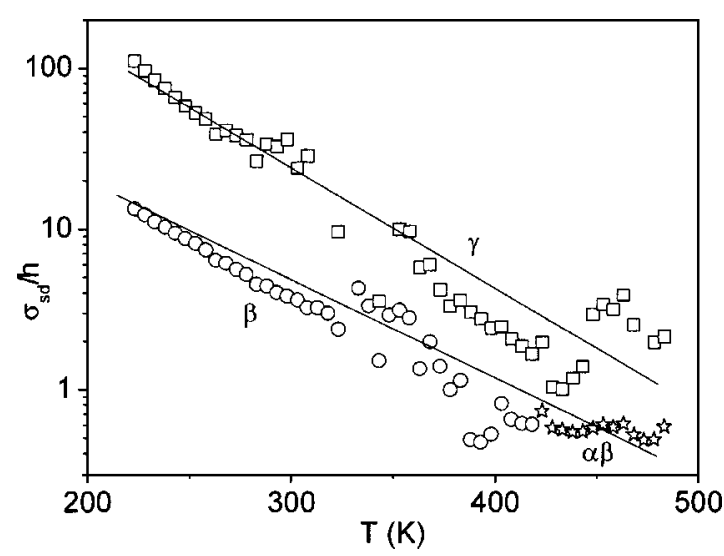

FIG. 7. Ratio of the standard deviation $\sigma_{\text {sd }}$ to the height $h$ of the $\beta(\bigcirc), \gamma$ $(\square)$, and ( 2 ) $\alpha \beta$ peaks of the retardation time spectra plotted as a function of temperature.

carried out by mirroring the points with respect to the lines $\tau=\tau_{\max }$. This technique was also used to study the spectra above the merging temperature, and the results prove that the $\alpha \beta$ peak is symmetric (Fig. 6). Subtracting the previously obtained symmetric peaks from the spectra caused deconvolutions of the $\alpha$ peaks. The normalized width of each of the $\beta$ and $\gamma$ peaks was obtained from the ratio of its standard deviation $\sigma_{\mathrm{sd}}$ to its height $h$. The results of this analysis, collected in Fig. 7, quantify the increase in both the narrowness and height of peaks as temperature increases. The strengths $\Delta \varepsilon$ of the $\alpha, \beta, \alpha \beta$, and $\gamma$ relaxations were obtained by quadrature, and the pertinent results over a wide temperature window are shown in Fig. 8. It can be seen that the strengths of the $\beta$ and $\gamma$ absorptions remain nearly constant in the glassy state, but they undergo a significant increase in the rubbery state. The strength of the $\alpha$ relaxation undergoes a relatively sharp decrease in a rather narrow temperature window, though the total dielectric strength above $T_{g}$ seems to be nearly independent on temperature.

The merging of the primary $\alpha$ relaxation and the secondary $\beta$ absorption has attracted the attention of many researchers in recent years because it has been related to the onset of the cooperativity of the $\alpha$ relaxation and also to the critical temperature $T_{c}$ of the mode-coupling theory. ${ }^{27,28}$ This latter

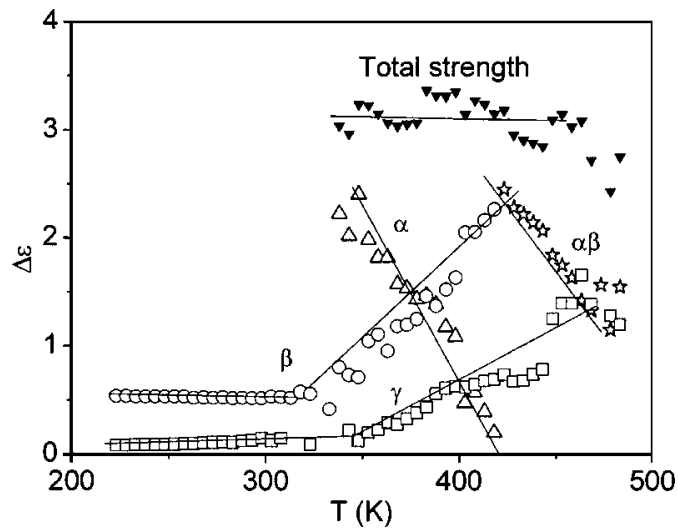

FIG. 8. Dependence of the strengths of the $\gamma(\square), \beta(\bigcirc), \alpha(\triangle)$, and $\alpha \beta$ (六) relaxations on temperature. Solid symbols read for the total strength, i.e., for the sum of the strengths of the four processes. The lines are only eye guides. 


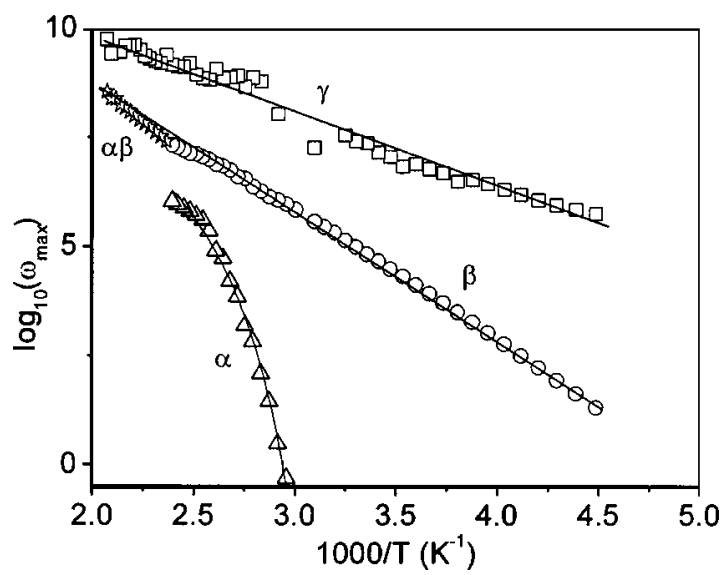

FIG. 9. Temperature dependence of the frequencies of the maxima $\omega_{\max }$ of the $\gamma(\square), \beta(\bigcirc), \alpha(\triangle)$, and $\alpha \beta$ (㐫) relaxations.

parameter was also associated with $T_{x}$, the temperature below which the energy landscape picture of the glass transition was proposed to be valid. ${ }^{29,30}$ The presence of the $\gamma$ absorption indicates that polymers with complex relaxation behavior such as PAMMD may not display a single $\alpha \beta$ absorption at temperatures well above $T_{g}$.

\section{Temperature dependence of the relaxations and the ionic conductivity}

Arrhenius plots for the $\alpha, \beta, \alpha \beta$, and $\gamma$ relaxations are shown in Fig. 9. The $\beta$ and $\gamma$ absorptions follow Arrhenius behavior with activation energies of about 11.3 and $8.5 \mathrm{kcal} \mathrm{mol}^{-1}$, respectively. The activation energy of the $\beta$ process is similar in the glassy and rubbery states and it is also similar to that of the $\alpha \beta$ process, this last fact suggesting that the latter relaxation was just a simple extension of the former. It is worth noting that the $\alpha$ relaxation vanishes before the Vogel curve [Eq. (1)] intersects the Arrhenius plot corresponding to the $\beta$ relaxation. Possible scenarios for the merging of the $\alpha$ and $\beta$ absorptions are described in the literature. $^{31,32}$

The relaxation time associated with the $\alpha$ absorption is also described by the Doolittle equation given by ${ }^{33}$

$$
\tau=A \exp \left(\frac{B}{\phi}\right),
$$

where $\phi$ is the free-volume fraction and $B$ is a parameter defined as ${ }^{34}$

$$
B=\kappa v^{*} / v_{m} .
$$

In this expression $\kappa$ is a constant lying in the interval 1-1.7, while $v^{*}$ and $v_{m}$ represent, respectively, the critical volume for a relaxation process to take place and the volume of the relaxing segments. By comparing the VFTH and the Doolittle equations the following expression for the free volume $\phi_{g}$ at $T_{g}$ is obtained

$$
\frac{\phi_{g}}{B}=\frac{T_{g}-T_{v}}{m} \text {. }
$$

Since the value obtained for $m$ from the Vogel $\ln \omega_{\max ; \alpha}$ versus $1 /\left(T-T_{v}\right)$ plot is $1840, \phi_{g} / B$ amounts to 0.027 , a

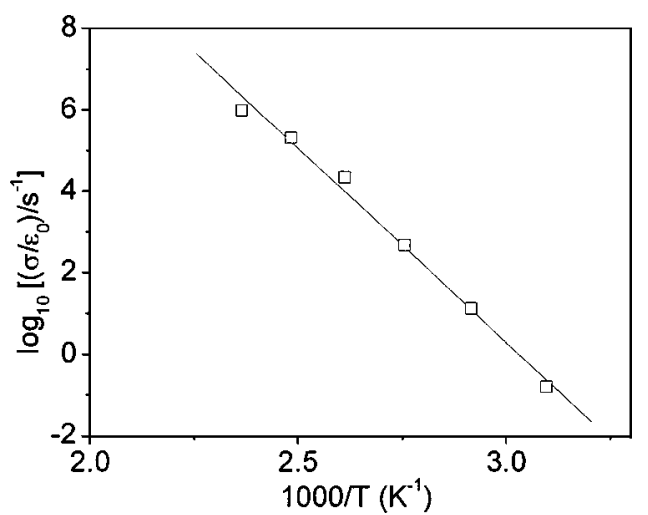

FIG. 10. Ratio of the ionic conductivity in Siemens $\sigma$ to the vacuum permittivity $\varepsilon_{0}$ plotted as a function of the reciprocal of temperature.

quantity which lies within the interval $0.025 \pm 0.005$ reported for most flexible polymers. ${ }^{35}$ Moreover, the thermalexpansion coefficient is $\alpha_{f}=(1 / v)(\partial v / \partial T)_{p} \cong 1 / m=5.4$ $\times 10^{-4} \mathrm{~K}^{-1}$, a value similar to that experimentally found for most amorphous polymers. ${ }^{35}$

The temperature dependence of the ionic conductivity expressed in terms of $\sigma / \varepsilon_{0}$ is shown in Fig. 10. It can be seen that the conductivity obeys Arrhenius behavior, with activation energy of the order of $33.1 \mathrm{kcal} \mathrm{mol}^{-1}$. Therefore ionic transport overcomes energy barriers more than three times higher than those involved in the $\beta$ relaxation.

\section{DISCUSSION}

Williams ${ }^{16,36}$ views the $\beta$ relaxation as caused by partial relaxation via limited local motions of dipolar groups that find themselves in a range of local environments. This limited relaxation leads to an initial fall in the time-dipole correlation function $\phi(t)$. At longer times, micro-Brownian motions cause fluctuations of local environments that lead to the total randomization of the dipole vector and as a result $\phi(t)$ becomes zero. Hence, the time-dipole correlation function can be written as

$$
\phi(t)=\phi_{\alpha}(t)\left[\sum_{r} p_{r} q_{r}+\phi_{\beta}(t) \sum_{r}\left(1-q_{r}\right)\right],
$$

where $\phi_{\alpha}(t)$ and $\phi_{\beta}(t)$ are, respectively, the time-dipole correlation functions for the $\alpha$ and $\beta$ relaxations, $p_{r}$ is the probability of obtaining environment $r$, and $q_{r}=\left\langle\mu_{r}\right\rangle^{2} /\left\langle\mu^{2}\right\rangle$, where $\left\langle\mu_{r}\right\rangle$ is the net dipole moment residing in the environment $r$ when the $\beta$ process has been completed and $\left\langle\mu^{2}\right\rangle$ is the mean-square dipole moment of the system. The $\alpha$ and $\beta$ relaxations are coupled phenomena because all of them presumably involve motions of the dipoles of the side group. In most cases, a small fraction of $\left\langle\mu^{2}\right\rangle$ is relaxed through the $\beta$ relaxation and the rest through the $\alpha$ relaxation. If $T$ $\gg T_{g}, \phi_{\alpha}(t)$ decays much faster than $\phi_{\beta}(t)$ and $\phi(t)$ decays via the $\alpha$ process. Therefore, the $\alpha \beta$ relaxation in many systems may be considered a continuation of the $\alpha$ process, with the difference that the $\alpha$ relaxation relaxes a part of $\left\langle\mu^{2}\right\rangle$ while the $\alpha \beta$ relaxation nearly relaxes the whole of $\left\langle\mu^{2}\right\rangle$. Although Eq. (22), also called Williams ansatz, holds for a variety of systems, it does not explain the findings of this 
work. ${ }^{37-39}$ In PAMMD, the $\alpha$ relaxation vanishes at high temperatures, and the $\alpha \beta$ relaxation is symmetric like the $\beta$ relaxation. It happens as whether with increasing temperature the $\alpha$ relaxation fed the $\beta$, until its eventual disappearance. To explain this behavior we could follow the approach of Stillinger, ${ }^{1}$ which assumes the configurational space above $T_{g}$ as formed by craters and small basins, the latter located not only in the space between craters but also inside the craters. The $\alpha$ and $\beta$ relaxations would take place across the depth craters and the basins, respectively. As temperature decreases, the craters become rare until a temperature is reached at which the $\alpha$ relaxation cannot occur, with only the basins remaining through which the $\beta$ process takes place. At high temperatures the depth of the craters in the configurational space presumably decreases, coming close to that of the basins. This would explain the similarity between the $\beta$ and $\alpha \beta$ processes.

The molecular mechanism behind the $\beta$ relaxation of poly(methylmethacrylate) is postulated to be the hindered rotation of the ester moiety about the $\mathrm{C}-\mathrm{C}(\mathrm{O})$ bond that connects it to the main chain, though involving some sort of intramolecular cooperativity. ${ }^{40}$ The polarization arising from the electric dipole associated with the carboxyl group relaxes through the $\beta$ relaxation and only a smaller part through the main chain dynamics reflected in the $\alpha$ relaxation. As a result this polymer exhibits a very strong $\beta$ process and a rather weak $\alpha$ relaxation. Replacing the methyl group of the ester moiety of the repeating unit of poly(methylmethacrylate) by a hydrogen atom enhances the conformational versatility of the resulting polymer, poly(methylacrylate). ${ }^{41}$ In this polymer, the polarization relaxes through the $\beta$ relaxation and also a significant part through segmental motions associated with the $\alpha$ relaxation. The complexity of the molecular motions of the side chains is greatly enhanced by the replacement of a hydrogen atom of the methyl group of poly(methylacrylate) with the 5-methyl-1,3-dioxacyclohexane moiety. ${ }^{9-11}$ Since the $\mathrm{C}(\mathrm{O})-\mathrm{O}$ bond of the ester group is restricted to the trans state, dielectric activity may arise from motions about the $-\mathrm{CH}_{2} \mathrm{C}(\mathrm{O}) \mathrm{O}-\mathrm{CH}_{2}-\mathrm{C}_{y}$ bonds coupled with both chair-to-inverse chair-conformational transitions occurring in the methyl-1,3-dioxcyclohexane ring and local motions of the skeletal bonds. The barrier energy involved in the conformational transition of the dioxacyclohexane ring has been calculated using molecular-dynamics simulation methods of 5,5-dimethyl-1,3-dioxacyclohexane. The energy associated with chair-to-inverse chair-conformational transition is nearly $9.7 \mathrm{kcal} \mathrm{mol}^{-1}$, close to the experimental activation energy of $11.3 \mathrm{kcal} \mathrm{mol}^{-1}{ }^{42,43}$ Accordingly, this transition accompanied with generalized motions of the side groups, presumably coupled with local motions of the main chain, may be held responsible for the ostensible $\beta$ relaxation exhibited by the dielectric spectra of PAMMD. The disappearance of the $\alpha$ relaxation at high temperatures suggests that $\left\langle\mu^{2}\right\rangle$ exclusively relaxes through the $\beta$ process. As for the $\gamma$ relaxation, it may arise from conformational transitions about $\mathrm{C}-\mathrm{C}(\mathrm{O})$ bonds characterized by having rather low torsional barrier energy.

\section{CONCLUSIONS}

The $\alpha$ relaxation vanishes before the curves describing the temperature dependence of the $\alpha$ and $\beta$ relaxations of poly(5-acryloxymethyl-5-ethyl-1,3-dioxacyclohexane) could intersect. Above $T_{g}$, the $\beta$ process of PAMMD increases at the expense of the $\alpha$ relaxation. The symmetry of the $\alpha \beta$ relaxation in conjunction with the fact that the Arrhenius behavior is common for the $\alpha \beta$ and $\beta$ relaxations suggests that both processes may have the same nature. The relaxation behavior of the polymer can be explained by assuming the existence of craters and basins in the potential-energy landscape, the basins being present even in the craters. The $\alpha$ relaxation arises from configurational transitions between craters, whereas the $\beta$ process presumably comes from transitions between basins. The density of craters depends on temperature, nearly vanishing in the vicinity of $T_{g}$, while the depth of the craters at high temperature may not significantly differ from that of the basins, this behavior explaining the similarity between $\beta$ and $\alpha \beta$ relaxations in PAMMD. The $\gamma$ relaxation might arise from transitions between sub-basins existing in the basins.

\section{ACKNOWLEDGMENTS}

The authors thank Dr. T. A. Ezquerra for helpful comments and for allowing us to use the dielectric experimental devices of his laboratory. We also thank Professor J. Guzmán for providing the polymer sample used in this work.

${ }^{1}$ F. F. Stillinger, Science 267, 1924 (1995)

${ }^{2}$ C. A. Angell, Science 267, 1935 (1995).

${ }^{3}$ H. Z. Vogel, Phys. Z. 22, 645 (1921)

${ }^{4}$ J. S Fulcher, J. Am. Ceram. Soc. 8, 839 (1925).

${ }^{5}$ G. Tammann and W. Z. Hesse, Z. Anorg. Allg. Chem. 156, 245 (1926).

${ }^{6}$ E. Donth, Relaxations and Themodynamics in Polymers: Glass Transition (Akademie, Berlin, 1992).

${ }^{7}$ P. Lukenheimer, U. Schneider, R. Brand, and A. Lidl, Contemp. Phys. 41, 15 (2000).

${ }^{8}$ J. Heijboer, Ph. D. thesis, University of Leiden, 1972.

${ }^{9}$ Y.-N. Huang, E. Saiz, T. Ezquerra, J. Guzmán, and E. Riande, Macromolecules 35, 2926 (2002).

${ }^{10}$ V. Compañ, R. Díaz-Calleja, J. Guzmán, and E. Riande, Polymer 42, 4339 (2001).

${ }^{11}$ R. Pérez-Feito, R. Díaz-Calleja, E. Riande, and J. Guzmán, J. Polym. Sci., Part B: Polym. Phys. 37, 2486 (1999).

${ }^{12}$ T. A. Ezquerra, G. Kremer, and G. Wegner, Progress in Electromagnetic Research, in Dielectric Properties of Heterogeneous Materials, edited by A. Priou (Elsevier, Amsterdam, 1992), Vol. 6.

${ }^{13}$ R. Fuoss and J. G. Kirkwood, J. Am. Chem. Soc. 63, 385 (1941).

${ }^{14}$ R. Kohlrausch, Ann. Phys. 12, 3931 (1847).

${ }^{15}$ G. Williams and D. D. Watts, Trans. Faraday Soc. 66, 80 (1970).

${ }^{16}$ G. Williams, in Keynote Lectures in Selected Topics of Polymer Science, edited by E. Riande (CSIC, Madrid 1995).

${ }^{17}$ G. Williams, D. C. Watts, S. B. Dev, and M. A. North, Trans. Faraday Soc. 67, 1323 (1971)

${ }^{18}$ S. Havriliak, Jr. and S. J. Havriliak, Dielectric and Mechanical Relaxation in Polymers (Hanser, New York, 1997).

${ }^{19}$ F. Álvarez, A. Alegría, and J. Colmenero, Phys. Rev. B 44, 7306 (1991); 47, 125 (1993).

${ }^{20}$ S. K. Kumar, R. H. Colby, S. H. Anastasiadis, and G. Fytas, J. Chem. Phys. 105, 3777 (1996)

${ }^{21}$ R. Díaz-Calleja, Macromolecules 33, 8924 (2000).

${ }^{22}$ E. Riande and R. Díaz-Calleja, Electrical Properties of Polymers (Marcel Dekker, New York, 2004).

${ }^{23}$ W. H. Press, S. A. Teukolsky, W. T. Vetterling, and B. P. Flannerty, Numerical Recipes in C: The Art of Scientific Computing, 2nd ed. (Cambridge University Press, New York, 1992). 
${ }^{24}$ V. A. Morozov, Methods for Solving Incorrectly Posed Problems (Springer, New York, 1984).

${ }^{25}$ P. C. Hansen and D. P. O'Leary, SIAM J. Sci. Comput. (USA) 14, 1487 (1993).

${ }^{26}$ S. W. Provencher, Comput. Phys. Commun. 27, 229 (1982).

${ }^{27}$ W. Gözte and L. Sjögren, Transp. Theory Stat. Phys. 24, 201 (1995).

${ }^{28}$ W. Gözte and L. Sjögren, Rep. Prog. Phys. 55, 241 (1992).

${ }^{29}$ M. J. Goldstein, Chem. Phys. 51, 3728 (1969).

${ }^{30}$ C. A. Angell, Proceedings of the XIV Sitges Conference: Complex Behavior of Glassy Systems, Sitges, Barcelona, Spain, June 1996, edited by M. Rubi and C. Pérez-Vicente (Springer, Berlin, 1997), Vol. 49, p. 1.

${ }^{31}$ F. Garwe, A. Schönhals, H. Lockwenz, M. Beiner, K. Schröter, and E Donth, Macromolecules 29, 247 (1996).

${ }^{32}$ A. Schönhals, in Dielectric Spectroscopy of Polymeric Materials: Fundamentals and Applications, edited by J. P. Runt and J. J. Fitzgerald (ACS, Washington, DC, 1997).

${ }^{33}$ A. K. Doolittle and D. B. Doolittle, J. Appl. Phys. 28, 901 (1957).

${ }^{34}$ M. H. Cohen and D. J. Turnbull, Chem. Phys. 30, 748 (1959).
${ }^{35}$ J. D. Ferry, Viscoelastic Properties of Polymers, 2nd ed. (Interscience, New York, 1972), p. 316.

${ }^{36}$ G. Williams, Adv. Polym. Sci. 33, 60 (1979).

${ }^{37}$ K. Schrötter, R. Unger, S. Reissig, F. Garwe, S. Kahle, M. Beiner, and E. Donth, Macromolecules 31, 8966 (1998).

${ }^{38}$ M. Beiner, S. Kahle, E. Hempel, K. Schröter, and E. Donth, Macromolecules 31, 8973 (1998)

${ }^{39}$ D. Gómez, A. Alegría, A. Arbe, and J. Colmenero, Macromolecules 34, 503 (2001)

${ }^{40}$ R. Bergman, F. Alvarez, A. Alegría, and J. Colmenero, J. Chem. Phys. 109, 7546 (1998).

${ }^{41}$ N. G. McCrum, B. E. Read, and G. Williams, Anelastic and Dielectric Effects in Polymeric Solids (Dover, New York, 1991), p. 286.

${ }^{42}$ E. Saiz, J. Guzmán, M. T. Iglesias, and E. Riande, J. Phys. Chem. 100, 18345 (1996).

${ }^{43}$ E. Saiz, E. Riande, R. Díaz-Calleja, and J. Guzmán, J. Phys. Chem. 101, 10949 (1997). 